dure to be used without providing nuclear medicine facilities. The simplicity of the technique enables it to be performed at the bedside at any time by anyone with a minimum of training in the use of radioisotopes and with the least inconvenience to the patient.

Since ${ }^{131}$ I-MAA was initially described as a lung scintiscanning agent, it is ideal for extension into a formal lung scan, permitting evaluation of perfusion deficiencies in cases of suspected pulmonary emboli.

Nuclear medicine is an expensive pastime, but the procedure, as we have described it, demands little in expertise, special skills, or manpower. In comparison with other nuclear medicine investigations it is inexpensive. It could become widely available but has the disadvantage of requiring pretest blockade of thyroidal uptake by sodium iodide. Sensitivity $(88 \%)$ and specificity $(91 \%)$ are comparable to those of similar techniques for detecting deepvein thrombosis. Even with this technique, however, the information yielded cannot be considered on a par with that of conventiona $x$-ray phlebography. Although we have not undertaken any clinical studies, there would appear to be no objection to using ${ }^{125}$ I-labelled MAA, with the consequent lowering of radiation dose to the patient. At the time of the original work ${ }^{125}$ I-MAA had not become available.

Denis D'Auria

Environmental and Medical Sciences

Division,

AERE Harwell,

' D'Auria, D, et al, British fournal of Surgery, 1973, 60 908.

Webber, M M, Webb, R, jun, and Craggin, M,
Medical Radioisotope Scintigraphy. 2, p 773, Medical Radioisotope
Vienna, IAEA, 1969.

\section{Driving after anaesthetics}

SIR,-As an anaesthetist who has volunteered many times for brief experimental nitrous oxide and oxygen anaesthetics, and who has followed up 81 outpatients anaesthetised for orthopaedic procedures, ${ }^{1}$ I would like to add a few observations to the discussion about residual effects of general anaesthetics (10 and 31 March, pp 686 and 891).

Subjective and objective changes in mood and behaviour induced by nitrous oxide may outlast retention of appreciable quantities of this gas. For example, a tendency to hypoxaemia induced by nitrous oxide in the lunch hour may be commented on by one's spouse in the evening, without knowledge of the precipitating circumstances. When nausea has resulted malaise has persisted. A lunchtime inhalation of $80 \%$ nitrous oxide with oxygen only up to the point of no response to repeated auditory stimuli may leave the volunteer subjectively aware of slight difficulty in assessing traffic speed while waiting at a $T$ junction in the evening. Although residual effects were not noticed 24 hours after anaesthesia, the fact that they appeared to outlast the presence of the causative agent should warn against simplistic predictions of recovery.

Nine of the 81 patients felt nauseated or actually vomited after outpatient anaesthesia and it is noteworthy that this was often precipitated by the journey home. Two patients felt nauseated or "sickly" for about two to three days. Two patients felt very tired for 24 hours.

If anyone takes up Dr Ogg's suggestion of a study of volurteers or patients subjected to driving tests at 24 and 48 hours after anaesthesia it might help to design driving tests to deliberately stimulate the semicircula canals, and to seek subjects who have a low threshold to general anaesthetics and who are easily nauseated. Duration of the driving test may also be relevant.

University Department of Anaesthesia,

W D A SMITH

Leeds LS2 9LN

'Smith, W D A, Outpatient anaesthesia with particula reference to nitrous oxide, appent

\section{Sex determination and the $\mathrm{H}-\mathrm{Y}$ antigen}

SIR,-Your lucid and comprehensive leading article (17 March, p 704) on H-Y antigen nevertheless omits certain evidence from one crucial abnormality of sexual development. As you mention, $\mathrm{H}-\mathrm{Y}$ antigen is expressed in true hermaphrodites, yet by definition such patients have ovarian as well as testicular tissue Moreover, the two types of gonads exhibit lateralisation, testes being preferentially situated on the right and ovaries on the left side. ${ }^{12}$ This asymmetry is strong evidence in favour of the involvement of an epigenetic factor in the gonadal differentiation of these patients. Clearly, if this epigenetic factor could be pinpointed, this might be expected to throw light on the general problem of gonadal differentiation.

A useful pointer comes from an observed asymmetry in the development of normal gonads. In human fetuses the two gonads differ in size and cell number, those on the right being on average somewhat larger, and containing more cells, than left-hand gonads. ${ }^{3}$ Furthermore, testes are markedly larger than ovaries in fetuses of similar crown-rumplength. ${ }^{3}$ If we make the assumption that fast growth is a prerequisite for testicular differentiation, the asymmetrical distribution of testes and ovaries in hermaphrodites is simply explained: testes tend to develop on the right because the gonad on this side is naturally faster growing than its partner on the left, and vice versa for the preferential location of ovaries on the left.

If we make the further assumption that the expression of $\mathrm{H}-\mathrm{Y}$ antigen is normally confined to the sex whose gonads grow faster than those of the other sex, another puzzling finding is explained. As mentioned in your editorial, $\mathrm{H}-\mathrm{Y}$ antigen is expressed in the males of mammals and is associated with testicular development, but in birds the antigen is expressed in females and is likely to be associated with ovarian development. What, then, is the underlying similarity between testicular development in mammals and ovarian development in birds? When we compare the relative growth rates of testes and ovaries, it becomes apparent that whereas mammalian testes grow faster than ovaries, in chick embryos aged 8 days onwards the ovaries grows faster than the testes. ${ }^{4}$ This difference in relative growth rates finds a parallel in the fact that in mammals the testis is the dominant embryonic gonad in contrast to birds, in which the ovary is the dominant embryonic gonad. ${ }^{6}$

The association of $\mathrm{H}-\mathrm{Y}$ antigen with the fast growing, dominant embryonic gonad provides a simple explanation for the finding that the antigen is expressed by the heterogametic sex regardless of whether this is the male, as in mammals, or the female, as in birds. It does not provide an answer to the chicken-and-egg question, whether the antigen causes gonadal differentiation or vice versa Whatever the precise relationship it is clear, however, that the antigen is associated not with any particular type of gonadal differentiation but with a common denominator underlying the development of mammalian testes and avian ovaries. ${ }^{7}$ There appears to be strong evidence that this common denominator is a relative effect which becomes apparent in a measurable difference in growth rate.

Ursula MitTwoch

Department of Genetics,

Lniversity College

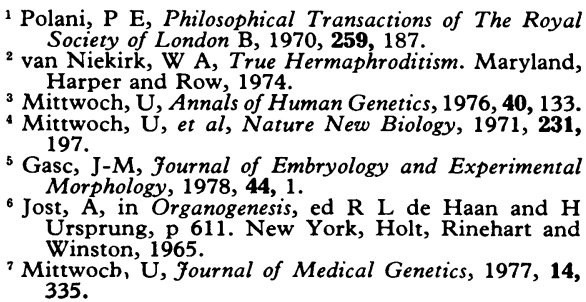

Polyunsaturated fatty acids in multiple sclerosis

SIR,-Dr D Bates and others (10 March, p 683) have had the misfortune to invest much time and effort in a clinical trial (18 November, p 1390) which, so far as $\alpha$-linolenate is concerned, is entirely nugatory. They did, however, from time to time receive verbal warnings that the prostaglandin $\mathrm{E}_{2}\left(\mathrm{PGE}_{2}\right)$ reaction produced by prolonged exhibition of (tartrazine-coated) Naudicelle capsules was puzzlingly out of step with those given by linoleic and arachidonic acids (LA and AA).

The circumscribed use of the drug advocated in my letter (10 February, p 411) and the tentative suggestion made earlier with respect to children ${ }^{2}$ surely do not merit the epithets "avowed propagandist for the use of Naudicelle" and "advocate of its administration to children." I plead guilty only to putting forward a new idea.

The electrophoretic tests used ${ }^{1}{ }^{2}$ have been validated against widely accepted indicators of lymphocyte sensitisation. ${ }^{34}$ Failure to "catch on" in "other laboratories in Britain" is immaterial since the method currently receives scholarly and unprejudiced study in the United States, West and East Germany, Italy, France, and other countries. Three international meetings are scheduled over the next six months to discuss the fully automatic and advanced laser-Doppler machines developed recently for simplified objective measurements.

Bates et al write in their paper that "the frequency of attacks was not significantly different between the four groups (table II)" (a difference from the original findings of Miller et $a l^{5}$ ), though when the "attack score" is used (a statistic of their own, inadequately explained) a "significant difference" emerges between columns $\mathrm{C}$ and $\mathrm{D}$. The deterioration of Naudicelle patients faster than controls $(P<0.05)$ is rejected by the authors as not biologically significant. I agree. But what is the $P$ value of the difference between $C$ and $D$ -not given in the statistical analysis?

A retest with pure $\alpha$-linolenate and adequate laboratory control throughout is needed. We know that some of the patients of Bates et al 